\title{
Inter-Core Crosstalk Impact on Migration Planning from Elastic Optical Networks to Spectrally-Spatially Flexible Optical Networks
}

\author{
Piotr Lechowicz $^{1}$, Rubén Rumipamba-Zambrano ${ }^{2}$, Jordi Perelló ${ }^{2}$, Salvatore Spadaro ${ }^{2}$ Krzysztof Walkowiak $^{1}$ \\ ${ }^{1}$ Wroclaw University of Science and Technology, Faculty of Electronics, Wybrzeże Wyspianskiego 27, 50-370 Wroclaw, Poland \\ Email: Piotr.lechowicz@pwr.edu.pl \\ ${ }^{2}$ Universitat Politècnica de Catalunya (UPC), Barcelona, Spain
}

\begin{abstract}
We study inter-core crosstalk (ICXT) impact on migration planning toward spectrallyspatially flexible optical networks considering 22-core multi-core fiber. Results reveal that 55$70 \%$ of links need to be upgraded when ICXT ranges from -68.2 to $-43.4 \mathrm{~dB} / \mathrm{km} .{ }^{1}$

OCIS codes: (060.0060) Fiber optics and optical communications; (060.4250) Networks.
\end{abstract}

\section{Introduction}

Spectrally-spatially flexible optical networks (SS-FONs), which combine flex-grid (elastic) networks (EONs) with space division multiplexing (SDM), are promising solution to overcome the capacity crunch problem. The key components required to realize SS-FONs are: i) SDM fibers, e.g. single-mode fiber bundles (SMFBs) or multi-core fibers (MCFs) and, ii) SDM-capable Reconfigurable Optical Add-Drop Multiplexers (SDM-ROADMs) [1]. One of the possible SDM-ROADMs assumed in the literature are broadcast-and-select fully non-blocking ROADMs supporting lane changes (B\&S FNB SDM-ROADMs [2,3]). One of the major concerns in MCFs is the effect of inter-core crosstalk (ICXT), i.e., the amount of signal leaking from adjacent cores to currently considered one, when transmitting signals in overlapping spectrum regions. After exceeding an acceptable crosstalk (XT) level, the proper reception of signal is impossible. Thus, the XT may significantly affect the transmission reach (TR) and thus the network performance [4]. In this paper, we focus on the impact of ICXT on migration planning from EONs to SSFONs. To this end, we evaluate several migration strategies and compare the migration performance between SMFBs and MCFs in terms of required number of replaced components.

\section{Transmission reach model}

As in [4], we estimate the acceptable lengths of routing paths when transmitting optical signals in an SDM network using MCF links, considering both the inter-core crosstalk effect and other linear and non-linear signal impairments. In this paper, we apply a worst-case XT estimation approach, which relies on evaluation of the maximum possible $\mathrm{XT}$ level that can occur in the fiber. In other words, the mean XT $\left(X T_{\mu}\right)$ level is calculated for the most affected fiber core, assuming that other connections are established on all adjacent cores. Worst-case XT increase per unit length of fiber $\left(X T_{d B, 1 \mathrm{~km}}\right)$ depends on several properties, such as number of adjacent cores, mode-coupling coefficient, propagation constant, bending radius and the core pitch [5]. The maximum transmission reach (TR) with regard to XT effect can be evaluated as follows:

$$
L_{\max , X T}=10^{\frac{X T_{d B, \max }-X T_{d B, 1 \mathrm{~km}}}{10}},(1) \quad L_{\max }=\min \left(L_{\max , X T}, L_{\max , P L I}\right),
$$

where $X T_{d B, \max }$ refer to the maximum XT limit. The authors of [6] provided acceptable values of XT for optical signals transmitted through weakly-coupled MCFs with different modulation formats (MFs). As in [4], we further decrease $X T_{d B, \max }$ by $7.7 \mathrm{~dB}$ according to [Hay], to account for stochastic XT changes and obtain 0.9999-quantile of the XT distribution. Therefore, the $X T_{d B, \max }$ is equal to -21.7, -26.2, -28.8 and -32.7 dB for BPSK, QPSK, 8QAM and 16-QAM MF, respectively. As the standards for MCF are yet expected (e.g., in [7], $X T_{d B, 1 \mathrm{~km}}=-56.2 \mathrm{~dB}$ ), we assume different values of $X T_{d B, 1 \mathrm{~km}}$ for $22 \mathrm{MCF}$, in particular, from $-68.2 \mathrm{~dB}$ to $-43.4 \mathrm{~dB}$, which are used to evaluate XT related transmission reaches using Eq. (1). However, in MCFs, other Physical Layer Impairments (PLIs) than XT may also affect signal transmission, and in consequence TR. To account for these PLIs, we apply model considered in [8], which provides the maximum TR of N-WDM super-channels with optical carriers occupying $37.5 \mathrm{GHz}$ and is equal to 6300, 3500, 1200 and $600 \mathrm{~km}$ for BPSK, QPSK, 8-QAM and 16-QAM MFs,

\footnotetext{
${ }^{1}$ The work of P. Lechowicz and K. Walkowiak was supported by National Science Centre, Poland under Grant 2015/19/B/ST7/02490 and statutory funds of Department of Systems and Computer Networks, Wroclaw University of Science and Technology. The work of R. Rumipamba-Zambrano is sponsored by SENESCYT, Ecuador. The work of J. Perelló and S. Spadaro was supported by the ALLIANCE project (TEC2017-90034-C2-2-R/C2-R).
} 
respectively. Finally, we find effective TR by taking the lower value of XT-related and other PLI-s related TRs for the assumed MCF parameters (Eq. (2)), which is presented in Fig. 1. Note, the XT-related PLIs starts to affect the TR for $X T_{d B, 1 \mathrm{~km}}$ higher than $-60 \mathrm{~dB}$. For more details, refer to [4].

\section{Network model and proposed solutions}

We assume that initially, the network deploys SMFs (as in EONs) and each node is equipped with legacy ROADMs. When all forecast traffic cannot be allocated, the need for enabling SDM transmission arises. To this end, each SMF links can be upgraded with SMFB- or MCF-enabled links and each legacy ROADM with, e.g., B\&S FNB SDMROADM [3]. The assumed SDM-ROADM is capable to direct any input fiber\&core to any output fiber\&core, i.e., it supports lane changes [8]. Transmission using SDM fiber is possible only when both connected nodes are SDMcapable. Otherwise, only one fiber/core in the SDM fiber can be utilized (as in EONs over SMFs) [2]. Each spatial mode (i.e., SMF in the SMFB, core in MCF) in the considered network provides $4 \mathrm{THz}$ bandwidth and applies flexible grid of $12.5 \mathrm{GHz}$ granularity. The network is equipped with coherent transceivers, each one operating at a fixed baud rate and transmitting/receiving an optical carrier (OC) that occupies $37.5 \mathrm{GHz}$ (3 frequency slices) [9]. The available modulation formats (MFs) are BPSK, QPSK, 8-QAM and 16-QAM supporting bit-rates of 50, 100, 150 and $200 \mathrm{Gbps}$, respectively. If the requested bit-rate exceeds a single transceiver capacity for the applied MF, the request is transmitted over several OCs using a set of adjacent slices (i.e., configuring a spectral SCh). In order to separate adjacent SChs, a fixed guard-band of $12.5 \mathrm{GHz}$ width is assumed. The supported TRs depend on the applied MF and the impact XT and are discussed in more details in Sec. II.

In this work, unicast and anycast flows are assumed. Unicast flows are one-to-one transmission technique for client requests, while anycast ones are defined as one-to-one-of-many transmission technique for communication between clients and the data centers (DCs). The DCs are spread geographically over the network and provide the same content. Therefore, for the client, it is insignificant which data center is used to provision the request. For simplicity, we assume that the connection to each DC is equivalent with the connection to the corresponding network node (the one connected to the DC), i.e., we do not consider the bandwidth limitation between DC and the backbone network. Moreover, the communication between DCs is also realized in the backbone network [10].

Regarding migration process, one critical decision emerges - which network node(s) and network link(s) should be upgraded to provision the whole forecast traffic. To this aim, we evaluate several strategies for nodes selection: i) the node with the highest degree (HND); ii) the node carrying the highest value of subcarriers (NCS); iii) the ending nodes of the link carrying the highest value of subcarriers (LCS). Nodes are selected and upgraded one-byone according to the applied strategy, until the whole traffic can be allocated in the network, i.e., at each step only one node is selected (or at most two in LCS). The fibers are replaced only when both end nodes connected to that link are already upgraded. When the number of spatially enabled fibers in the network increases, the traffic is reallocated. Note, at each step, only nodes still not upgraded are considered for selection.

In order to allocate the offered demands in the network, the k-shortest path first-fit method is used. At first, demands are sorted in descending order, according to the required number of subcarriers on their shortest path. Then, demands are processed consecutively, and for each one, all candidate paths are checked, and the request is provisioned using the lowest optical corridor found, i.e., the highest allocated slice in the network is minimized.

\section{Performance evaluation}

We evaluate our proposed solution in a national US network topology (26 nodes, 84 links) [2], considering two scenarios: i) replacement of SMFs with SMFBs; ii) replacement of SMFs with MCFs. Each SDM fiber comprises 22 spatial modes. As in [10], we assume that there are 7 DCs located in some network nodes, each one providing the same content. In most of the experiments, we assume that the number of candidate (shortest) routing paths for each demand is 10 , and $X T_{d B, 1 \mathrm{~km}}=-56.2 \mathrm{~dB}$ for MCF. For each path, we select the MF with the highest spectral efficiency supporting the required TR. The overall offered traffic volume is between 20 and 500 Tbps, with a 20 Tbps granularity, and for each value, we evaluate 10 randomly generated sets of demands. We consider three types of requests: i) city-to-city - unicast demand between any pair of network nodes; ii) city-to-dc - anycast demand between the client node and one of the available DCs; iii) dc-to-dc - unicast demand between any pair of DCs [10]. Each city-to-city and dc-to-dc demand has randomly selected source/destination nodes and a bit-rate uniformly selected within the range from $50 \mathrm{Gbps}$ to $1 \mathrm{Tbps}$ with $50 \mathrm{Gbps}$ granularity. Contrarily, each city-to-dc demand has randomly selected client node, direction, i.e., upstream or downstream, and a bit-rate uniformly selected within the range from $200 \mathrm{Gbps}$ to $1 \mathrm{Tbps}$ with $200 \mathrm{Gbps}$ granularity. The traffic proportion for city-to-city, city-to-dc, dc-todc traffic is $0.5,0.3,0.2$, respectively. We assume that the traffic is gradually increasing, and therefore, at each stage, only not yet migrated nodes and links may be upgraded. As the main performance metric, we take the ratio of upgraded nodes to all nodes (node migration ratio, abbr. NMR) and ratio of replaced links to all links in the network (fiber migration ratio, abbr. FMR) that allows for pending traffic allocation. 
The experiments are aimed at comparing performance differences between SMFB and MCF regarding migration planning. In Fig. 2 and 3, NMR and FMR, respectively, are presented in a function of offered total traffic for various selection strategies. The key observation is that LCS strategy outperformance two other ones - after migrating $70 \%$ of links to MCFs and $81 \%$ of nodes, a 15-fold traffic increase is achieved. Moreover, the difference of NMR (FMR, respectively) between SMFB and MCF for LCS strategy is equal to 5.38 (5.95) and 8.84 (12.62) percentage points (p.p.) for 200 and 400 Tbps offered traffic. All further results assume total traffic of 300 Tbps. As the next step, the impact of different values of $X T_{d B, 1 \mathrm{~km}}$ of 22-core MCF on the migration performance are evaluated. Fig. 4 presents NMR and FMR in function of $X T_{d B, 1 \mathrm{~km}}$ for various selection strategies. According to the results, the significant performance decrease is observed for $X T_{d B, 1 \mathrm{~km}}$ between -60 and $-54 \mathrm{~dB}$. In particular, to provide 15-fold traffic increase, $55-70 \%$ of links and 70-81\% of nodes need to be upgraded when ICXT ranges from -68.2 to $-43.4 \mathrm{~dB} / \mathrm{km}$. The differences in performance can be justified with Fig. 5, where percentage usage of MFs is reported in function of $X T_{d B, 1 \mathrm{~km}}$. As $T_{d B, 1 \mathrm{~km}}$ increases, less spectral efficient MFs are more often used, because the supported TRs are decreasing. Note, the differences in migration ratio between Fig. 2, 3 and 4 come from the fact that in further experiments only one value of traffic is considered, i.e., the traffic is not gradually increasing. Finally, Fig. 6 shows SMFB to MCF cost ratio, i.e., a ratio of fibers migration cost using SMFBs to MCFs, required to fulfill the offered traffic (300 Tbps). Parameter $\gamma$ denotes the ratio of deployment cost of one SMFB to one MCF. When SMFB to MCF ratio is greater 1, it denotes that it is more profitable to use MCFs. According to the results, for MCFs with $X T_{d B, 1 \mathrm{~km}}=-56.2 \mathrm{~dB}$, it is more profitable to use MCFs, when deployment of SMFBs is at least 1.2-fold more expensive than MCFs.

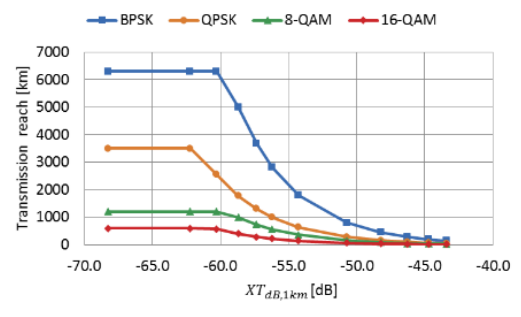

Fig. 1. Effective TR as a function of the worst-case inter-core XT in 22-core MCF.

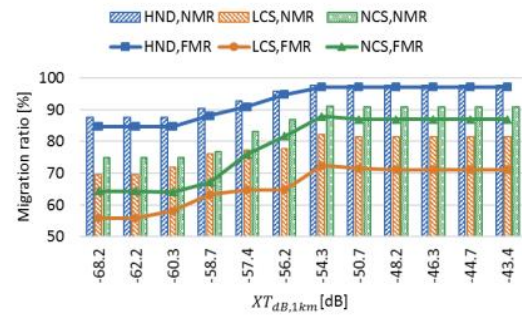

Fig. 4. NMR and FMR for MCF in a function of $X T_{d B, 1 \mathrm{~km}}$ for various strategies.

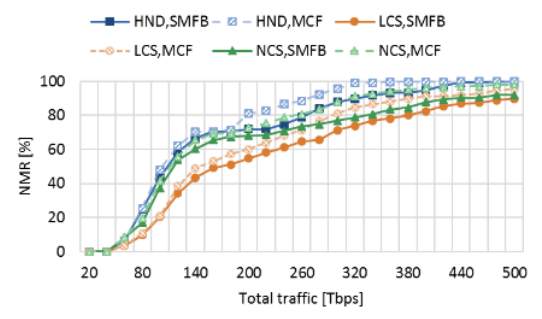

Fig. 2. NMR in a function of total traffic.

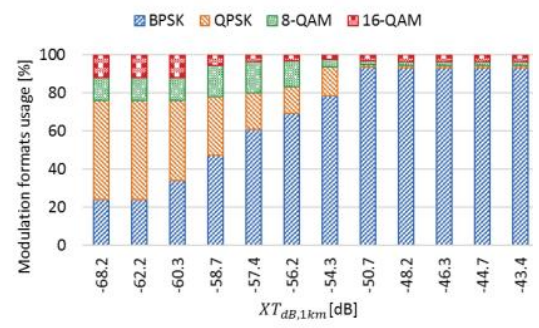

Fig. 5. MFs usage in a function of $X T_{d B, 1 \mathrm{~km}}$ for LCS strategy.

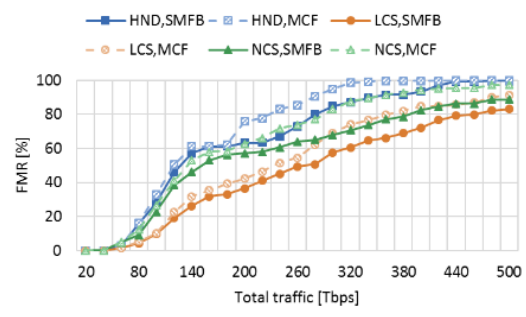

Fig. 3. FMR in a function of total traffic.

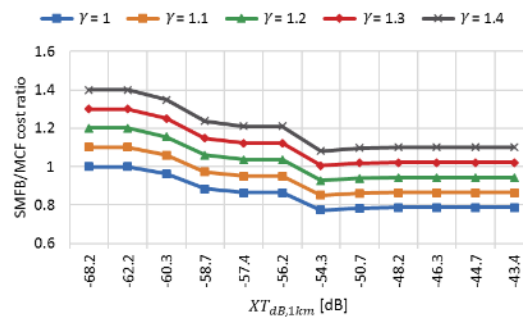

Fig. 6. Cost ratio between SMFB and MCF in a function of $X T_{d B, 1 \mathrm{~km}}$ for LCS strategy.

\section{Conclusions}

In this paper, we focus on the inter-core crosstalk impact on migration planning from EONs to SS-FONs applying either SMFBs or MCFs. According to the results, the 15-fold traffic increase can be achieved when migrating $70 \%$ links from SMFs to MCFs. Furthermore, different assumptions about $X T_{d B, 1 \mathrm{~km}}$ may have a significant impact on the network performance, in particular, when $X T_{d B, 1 \mathrm{~km}}$ is above $-60 \mathrm{~dB}$, it is required to migrate higher number of nodes and links. As the future work, we plan to develop metaheuristic algorithm for migration planning.

\section{References}

[1] D. Klonidis, et al. "Spectrally and spatially flexible optical network planning and operations," IEEE Com. Mag., 2015.

[2] P. Lechowicz et al., "Migration Planning from Elastic Optical Networks to Spectrally-Spatially Flexible Optical Networks," in Proc. of PSC, 2018

[3] R. Rumipamba-Zambrano et al.,"Assessment of Flex-Grid/MCF Optical Networks with ROADM limited core switching capability," ONDM, 2017

[4] M. Klinkowski et al., "A Study on the Impact of Inter-Core Crosstalk on SDM Network Performance", in Proc. of ICNC, 2018

[5] T. Hayashi et al., "Design and fabrication of ultra-low crosstalk and low-loss multi-core fiber," Optics Express, vol. 19, no. 17, 2011

[6] A. Muhammad et al., "Resource allocation for space division multiplexing: Optical white box vs. optical black box networking," IEEE JLT, 2015

[7] R. Rumipamba-Zambrano et al., "Capacity Quantification of Joint-switching-enabled Flex-Grid/SDM Optical Backbone Networks," in OFC 2017

[8] P. S. Khodashenas et al., "Comparison of spectral and spatial super-channel allocation schemes for SDM networks," IEEE JLT, 2016

[9] C. Rottondi et al., "Optical ring metro networks with flexible grid and distance-adaptive optical coherent transceivers," Bell Labs Tech. J., 2013.

[10] R. Goscien et al., "Distance-adaptive transmission in cloud-ready elastic optical networks," IEEE/OSA JOCN, 2014 